\title{
Capacidades emprendedoras en población de la base de la pirámide en Cúcuta, Colombia
}

\author{
Entrepreneurial capacities in population of the base of the pyramid in \\ Cúcuta, Colombia
}

\section{Neida Albornoz-Arias*, Julio César Contreras-Velásquez, Jhon Franklin Espinosa-Castro, Mauricio Enrique Sotelo Barrios}

Universidad Simón Bolívar, Colombia

Recibido el 4 de septiembre de 2018; aceptado el 7 de noviembre de 2019

Disponible en Internet el: 7 de noviembre de 2019

\section{Resumen}

El presente artículo estudia las capacidades emprendedoras en población de la Base de la Pirámide (BoP) en Cúcuta, Norte de Santander, Colombia. El objetivo fue determinar la relación entre la percepción de la habilidad para detectar y aprovechar oportunidades para la mejora de los resultados (variable dependiente) y las variables independientes: género, edad, nivel educativo, perfil ocupacional, pasión, visión, capacidad de aprendizaje, logro de resultados, determinación, creatividad e innovación, persistencia, trabajo en equipo, liderazgo, autoestima, asumir riesgos y organización. Se desarrolló bajo el paradigma positivista, enfoque cuantitativo, utilizando un muestreo no probabilístico. La muestra estuvo conformada por 197 emprendedores con edades entre 18 y 60 años. En la recolección de datos se empleó una encuesta estructurada y luego se hizo un análisis multivariante a través de una regresión categórica con escalamiento óptimo (CatReg). Las medidas de importancia relativa de Pratt, muestran la importancia de los predictores: 1) persistencia; 2) visión; 3) organización y 4) logro de resultados, aportan el $86.4 \%$ del modelo para explicar la variable dependiente. Se observa la ausencia de la variable creatividad e innovación, indispensable para un emprendimiento por oportunidad, sostenible en el tiempo; así como otras variables.

\section{Código JEL: L26, M13, P42}

Palabras clave: Emprendimiento; Capacidades emprendedoras; Base de la pirámide; Negocios inclusivos

* Autor para correspondencia

Correo electrónico n.albornoz@unisimonbolivar.edu.co (N. Albornoz Arias).

La revisión por pares es responsabilidad de la Universidad Nacional Autónoma de México. 


\begin{abstract}
This article studies the entrepreneurial skills in population of the Pyramid Base (BoP) in Cúcuta, Norte de Santander, Colombia. The objective was to determine the relationship between the perception of the ability to detect and take advantage of opportunities for improving results (dependent variable) and independent variables: gender, age, educational level, occupational profile, passion, vision, learning capacity, achievement of results, determination, creativity and innovation, persistence, teamwork, leadership, self-esteem, taking risks and organization. It was developed under the positivist paradigm, quantitative approach, using non-probabilistic sampling. The sample consisted of 197 entrepreneurs between the ages of 18 and 60. A structured survey was used in the data collection and then a multivariate analysis was performed through a categorical regression with optimal scaling (CatReg). Pratt's measures of relative importance show the importance of predictors: 1) persistence; 2) vision; 3) organization and 4 ) achievement of results, contribute $86.4 \%$ of the model to explain the dependent variable. We observe the absence of creativity and innovations variables, indispensable for an entrepreneurship for opportunity, sustainable over time; as well as other variables.
\end{abstract}

JEL code: $\mathrm{L} 26, \mathrm{M} 13, \mathrm{P} 42$

Keywords: Entrepreneurship; Entrepreneurial skills; Base of the pyramid; Inclusive businesses

\title{
Introducción
}

La base de la pirámide poblacional (BoP) está compuesta por los hogares con niveles socioeconómicos más bajos o personas pobres de cada país. Ellos son consumidores y emprendedores capaces de poner en marcha su propio negocio en el sector privado. Las fortalezas de este sector son por una parte el gran número de personas que lo conforman y que son emprendedores ingeniosos que se orientan por los bajos costos y por otra, la existencia de una importante proporción de pobres de bajos ingresos dispuestos a demandar bienes y servicios (tecnología, salud, alimentos, vivienda, créditos entre otros), generados por los emprendedores de este mismo sector (Prahalad, 2005). La BoP se encuentra desatendida en términos de soluciones estables en el tiempo (Contreras, Wilches, Delgado y Cerda, 2016), las políticas y programas asistencialistas, comúnmente son inútiles para llevar a los pobres hacia la clase media.

Desde la posición de consumidores, la BoP está representada aproximadamente por cuatro billones de personas y representan cinco trillones de dólares del mercado global del consumo. En América Latina, son 360 millones de personas en el setenta por ciento de la población según Hammond et al (2007). Por su parte, Azevedo et al (2015) indican que para 2010, esa cifra se ubicó en 400 millones de personas, ellos consideraron las encuestas 
nacionales de ingresos y gastos del hogar para realizar dicha estimación, señalando también que las personas que viven con menos de 10 dólares al día, se incrementó en $22 \%$ entre el 2000 y 2010, aumentando de 623.000 millones de dólares anuales en el año 2000 a 759.000 millones de dólares en 2010; que los consumidores de la BoP en América Latina y El Caribe han mejorado sus condiciones de vida en términos de ingresos, urbanismo, educación, acceso a las tecnologías de información y comunicación, debido al crecimiento económico de la región entre 2000 y 2010. De igual manera, 63\% del consumo de la BoP se ubica en los sectores alimentos (US\$209 mil millones anuales), vivienda (US\$184 mil millones anuales) y transporte (US\$82 mil millones anuales).

En cuanto a las experiencias empresariales en la BoP, se relacionan con la Responsabilidad Social Empresarial de importantes empresas, es decir, algunas han aprovechado este mercado como oportunidad de negocio, innovando y aprovechando el incremento en su cuota de mercado, a través de la internacionalización (Schuster y Holtbrügge, 2012). En el mundo, se destacan algunas multinacionales en los mercados de la BoP como son: Smart Communications en Filipinas y Vodafone en África mediante innovaciones tecnológicas; Nestlé en el 2006 con una inversión por el orden de US\$ 47 millones para instalar una fábrica empacadora y de distribución de productos con empaques más baratos para poder abastecer a mercados con menos poder adquisitivo, un mercado valuado en US\$2.800 millones a nivel global (Mutis y Ricart, 2008).

En Colombia, también existen iniciativas de empresas que han entrado en el mercado de la BoP como: Empresas Públicas de Medellín (EPM) orientada hacia la universalización de los servicios públicos; Promigás con un modelo de financiación no bancaria; Alpina con productos populares y Comfama con un modelo de arrendamiento social con apoyo del BID (RedE América, 2013).

Estas estrategias de importantes empresas dirigidas al mercado de la BoP son llamadas por Simanis y Hart (2009), estrategias de primera generación (BoP 1.0) conducentes en explotar comercialmente estos mercados. Estos autores propusieron las estrategias de segunda generación (BoP 2.0), determinadas a través de la interacción con gente local, para generar la invención y creación conjunta de las oportunidades de negocios.

Con relación a éstas últimas, en los últimos años se vienen fomentando los Negocios Inclusivos (NI), a través de modelos de negocios sostenibles enfocados en el mercado de la BoP, que empoderan a la población vulnerable, asociando sus necesidades socioeconómicas con la creación de trabajos y fuentes estables de ingresos (Pineda-Escobar, 2014), que incluyen a los pobres como clientes y consumidores (desde la demanda) y como empleados, productores y propietarios del negocio (desde la oferta) (PNUD, 2010); contribuyen positivamente al desarrollo de negocios, mejoramiento de las condiciones de vida de la población local y del medio ambiente (Gradl y Knobloch, 2010). 
Bajo el enfoque de los NI desde el lado de la oferta, en el presente estudio se abordaron las capacidades emprendedoras en población de la BoP en Cúcuta, Norte de Santander, Colombia, considerando que las mismas involucran elementos distintivos de un individuo. En este sentido, se plantea como pregunta de investigación ¿la percepción de la habilidad para detectar y aprovechar oportunidades para la mejora de los resultados tiene relación con el género, edad, nivel educativo, perfil ocupacional, pasión, visión, capacidad de aprendizaje, logro de resultados, determinación, creatividad e innovación, persistencia, trabajo en equipo, liderazgo, autoestima, asumir riesgos y organización?

Por lo tanto, el objetivo planteado es determinar la relación entre la variable dependiente la percepción de la habilidad para detectar y aprovechar oportunidades para la mejora de los resultados y las variables independientes: género, edad, nivel educativo, perfil ocupacional, pasión, visión, capacidad de aprendizaje, logro de resultados, determinación, creatividad e innovación, persistencia, trabajo en equipo, liderazgo, autoestima, asumir riesgos y organización.

El estudio se desarrolló bajo el paradigma positivista, enfoque cuantitativo, basado en un muestreo no probabilístico. Se empleó un tipo de muestro intencional. La muestra estuvo conformada por 197 emprendedores del mercado de la BoP de la ciudad Cúcuta, Norte de Santander, Colombia, personas con edades entre 18 y 60 años, pertenecientes principalmente a los estratos socioeconómicos 1 y 2 . En la recolección de datos se empleó una encuesta estructurada. Se utilizó el análisis multivariante a través de una regresión categórica con escalamiento óptimo (CatReg) con el propósito de describir, cuantificar y determinar las relaciones entre las variables predictores y la variable dependiente, así como determinar la influencia o importancia de estas relaciones sobre la variable dependiente.

\section{Capacidades emprendedoras}

El emprendimiento se relaciona con crecimiento económico y desarrollo en un país, cuando el mismo involucra la innovación; proceso para el cual también son necesarias las capacidades técnicas, intuición y estrategia del individuo y su equipo de trabajo. Este individuo es conocido como emprendedor, quien no necesariamente es inversionista, tampoco inventor o directivo, sino aquel que explota una invención, asigna recursos, toma decisiones y organiza la actividad económica en escenarios de incertidumbre (Schumpeter, 1961). A su vez, es motivado por factores internos y externos (motivación intrínseca y extrínseca), combina sus conocimientos, capacidades y habilidades para materializar una idea de negocio (Marulanda, Montoya y Vélez, 2014).

La motivación intrínseca es el interés individual que une el propósito y la acción del emprendedor y lo promueve a esforzarse, ser persistente, tomar decisiones (Eijdenberg y Masurel, 2013), es decir el individuo emprendedor posee ciertas capacidades para ser 
empresario. La persistencia se refiere a la cualidad de ser perseverante, constante, firme y decidido con el desarrollo del proyecto emprendedor que está desarrollando. La persistencia en el emprendedor debe perdurar si éste desea el crecimiento de su negocio, pues implica también sacrificios para superar dificultades del entorno (Bandura, 1986, 1989); preocuparse por incorporar la innovación en todo su proceso económico y por los estándares de calidad del servicio y producto, así como de la atención al cliente (Barrera, 2012).

La pasión la posee una persona cuando ama lo que hace y para amar lo que se hace, debe sentirse en el impulso del espíritu, le resulta fácil hacer las actividades del proyecto con placer y confianza (Freire, 2005). El emprendedor con visión posee una cualidad proactiva manifestada en una perspectiva de futuro, que le motiva a desarrollar nuevos productos o servicios o mejorar los actuales; interviniendo de manera anticipada frente a las oportunidades o cambios (Hughes y Morgan, 2007).

Emprender es un proceso cuyo resultado y éxito están determinados por la ideación y ejecución de mecanismos de aprendizaje, que no es otra cosa que el control sobre la cultura que contiene reglas simbólicas (Ries, 2011; Sarasvathy, 2001). Otra cualidad emprendedora es la necesidad de logro y se vincula con el impulso a sobresalir en comparación con un estándar (McClelland, 1961). El individuo está impulsado a trabajar por la excelencia (Robbins, 1999) e implica la constancia para trabajar día a día por lograr los objetivos planteados (López, Montilla y Briceño, 2007).

El emprendedor actúa con determinación cuando su motivación interior le impulsa a alcanzar una meta u objetivo, de manera voluntaria, con perseverancia y pasión; comprende la suma de la intención, ambición y disciplina (Grant-Halvorson, 2012); donde la persona actúa de manera planificada, diseñando un plan organizado con objetivos establecidos al momento de iniciar el plan de negocio (Martínez-Caro y Cegarra-Navarro, 2012). La tendencia del emprendedor hacia la creatividad e innovación es observada cuando éste manifiesta la voluntad para apoyar nuevas ideas, novedosas, de manera creativa con la finalidad de crear nuevos productos o servicios (Schumpeter, 1961; Lumpkin y Dess, 1996).

Las iniciativas para incrementar la tasa de actividad emprendedora pueden tener éxito a través de la educación (Oosterbeek, van Praag y IJsselstein, 2008), pues la formación en emprendimiento fomenta el autoestima y confianza en el emprendedor, además lo dota de herramientas para interactuar con el contexto y poner en marcha su negocio. La autoestima, se refiere a cómo una persona se siente acerca de sí mismo; cómo los pensamientos y sentimientos de valoración y aceptación que cada persona tiene de sí misma, cambia y es mejorable, aunque está influida por su motivación (Carreño et al, 2018; Rodríguez y Caño, 2012; Ferkany, 2008), concepto que sugiere que el emprendedor es una persona madura, se quiere a sí misma y es flexible adaptarse a los cambios frente a una situación que considere favorable para él.

El emprendedor debe tener la capacidad de ser parte de un colectivo y trabajar en cola- 
boración con otros, enfocado en lograr objetivos comunes que beneficien a algunos (Duarte y Tibana, 2009); para ello es indispensable que sea líder, que pueda influir en otras personas para que realicen sus tareas y lograr un objetivo común (Castellanos, Chávez y Jiménez, 2003); liderazgo que implica la capacidad para agrupar y organizar grupos de personas, cuya cooperación puede ser necesaria para la creación de grupos de trabajo y alianzas (Achig et al, 2018); también observa y aprovecha oportunidades a cada momento (Recalde, Villota y Flores, 2017).

La labor de explorar y descubrir nuevas oportunidades de negocio involucra para el emprendedor, adoptar conductas arriesgadas (implica desde perder el capital hasta el daño psicológico como frustración, decepción, pesimismo), especialmente cuando se desean mayores recompensas (Lumpkin y Dess, 1996; López, Montilla y Briceño, 2007).

El desarrollo y posesión de las capacidades emprendedoras referidas anteriormente responden al concepto de orientación emprededora $(\mathrm{OE})$, entendido como la generación de estrategia emprendedor que involucra visión y manejo de ventajas competitivas en el corto, mediano y largo plazo; con capacidad de innovación, para asumir riesgos, ser proactivo y agresivo con competidores, aprovechando oportunidades, por lo tanto se trata de personas con capacidad para sacar adelante un emprendimiento por oportunidad (Rodrigo-Alarcón, Parra-Requena y García-Villaverde, 2014).

\section{Metodología}

El estudio se desarrolló bajo el paradigma positivista, centrado en semejanzas y afirmaciones nomotéticas (Ricoy, 2006). La fase empírico-analítica se llevó a cabo a través de recolección de información suministrada por emprendedores del mercado de la BoP de la ciudad Cúcuta, Norte de Santander, Colombia, personas con edades entre 18 y 60 años, pertenecientes principalmente a los estratos socioeconómicos 1 y 2. Según el Departamento Administrativo Nacional de Estadísticas (DANE), en Colombia existen seis estratos socioeconómicos. En este caso el estrato 1: bajo-bajo y estrato 2: bajo; son aquellos que incluyen a la población con menores recursos y que son beneficiarios de subsidios en los servicios públicos domiciliarios.

Se empleó el enfoque cuantitativo. Dado que se desconocía el tamaño de la población, se utilizó un muestreo no probabilístico, el cual permite seleccionar los casos que aportan la mayor y mejor información del objeto de estudio, donde el investigador selecciona los casos que tiene más disponibles (Alaminos y Castejón, 2006). Se empleó un tipo de muestro intencional, que permite testar cuestionarios de nuevo diseño, no permite hacer ningún tipo de inferencia sobre la población y las conclusiones se refieren a la misma muestra (Alaminos 
y Castejón, 2006). La muestra estuvo conformada por 197 personas de los cuales $77.66 \%$ fueron mujeres y $22.34 \%$ hombres.

La técnica empleada para recolectar los datos fue la encuesta estructurada, que permitió normalizar la interacción del entrevistador y el entrevistado. El instrumento está divido en dos partes, la primera contiene diez preguntas sobre datos sociodemográficos y la segunda parte, diecisiete apartados relacionados con los ítems de las capacidades emprendedoras que se estudiaron. Se empleó una escala de Likert; pudiendo escalar a los sujetos en torno a sus capacidades emprendedoras; asumiendo un nivel de medida ordinal y considerando las siguientes alternativas de respuesta: 1-nunca, 2-pocas veces, 3-regularmente, 4-casi siempre, 5-siempre (Alaminos y Castejón, 2006).

El levantamiento de información primaria fue realizado en la ciudad de Cúcuta, capital del Departamento Norte de Santander, Colombia. Este Departamento cuenta con una población de 1.346.806 habitantes y la ciudad de Cúcuta con 703.000 y representa 52,1\% de la población total del Departamento. El 56\% de la población pertenece a los estratos socioeconómicos 1 y 2 (DANE, 2018).

El instrumento fue validado por tres expertos (un estadístico y dos expertos en emprendimiento). Antes de la aplicación fueron entrenados cinco encuestadores que apoyaron el levantamiento de información primaria. El instrumento fue aplicado en un único momento, cuando fue convocada la comunidad que habita en la comuna 7, del Barrio Simón Bolívar de la ciudad de Cúcuta, Colombia, para una actividad de gestión social de la Universidad Simón Bolívar, sede Cúcuta.

La percepción de habilidad para detectar y aprovechar oportunidades para la mejora de resultados es definida como variable dependiente. Según Osorio, Gálvez y Murillo (2010), el emprendedor afronta constantemente diversas fuerzas en el proceso emprendedor; fuerzas que provienen del entorno como es la oportunidad aprovechable en un proceso empresarial. Se trata de una habilidad del individuo para generar valor económico y social, donde el proceso de emprendimiento consiste en la búsqueda de una oportunidad central o estado futuro deseable, al incorporar bienes, materias primas, servicios entre otros elementos (Van der Veen y Wakkee, 2002). En la Tabla 1, se detallan las variables consideradas en el estudio y su respectiva escala de medida. 
Tabla 1

Características de las variables originales del estudio

\begin{tabular}{|c|c|c|c|c|c|}
\hline \multirow{3}{*}{$\begin{array}{l}\text { Variable } \\
\text { Dependiente }\end{array}$} & & & \multirow[b]{3}{*}{$\%$} & $95,0 \%$ & \multirow{3}{*}{$\begin{array}{r}95,0 \% \\
\text { CL superior } \\
\text { para } \%\end{array}$} \\
\hline & & Escala de & & CL inferior & \\
\hline & & Medida & & para \% & \\
\hline ¿Posee habilidad & & 1-Nunca & 0 & 0 & 0 \\
\hline para detectar & Categórica & 2-Pocas veces & 5.58 & 3.00 & 9.45 \\
\hline y aprovechar & Ordinal & & & & \\
\hline oportunidades & & 3-Regularmente & 11.68 & 7.76 & 16.71 \\
\hline \multicolumn{2}{|l|}{ para la mejora } & 4-Casi Siempre & 49.75 & 42.81 & 56.69 \\
\hline $\begin{array}{l}\text { de resultados? } \\
\text { (Sentido de Opor- } \\
\text { tunidad) }\end{array}$ & & 5-Siempre & 32.99 & 26.71 & 39.77 \\
\hline \multicolumn{6}{|l|}{$\begin{array}{l}\text { Variables } \\
\text { explicativas }\end{array}$} \\
\hline \multirow{4}{*}{ Género } & & 1-Femenino & 77.66 & 71.47 & 83.05 \\
\hline & Categórica & 2-Masculino & 22.34 & 16.95 & 28.53 \\
\hline & Nominal & & & & \\
\hline & & 18 a 25 años & 65.48 & 58.66 & 71.86 \\
\hline \multirow{2}{*}{ Edad } & Categórica & 26 a 35 años & 22.84 & 17.40 & 29.07 \\
\hline & Ordinal & & & & \\
\hline \multirow{7}{*}{ Nivel educativo } & & 36 a 50 años & 11.68 & 7.76 & 16.71 \\
\hline & & Primaria & 3.05 & 1.28 & 6.17 \\
\hline & Categórica & Bachillerato & 45.69 & 38.83 & 52.66 \\
\hline & Ordinal & & & & \\
\hline & & Técnico Laboral/ & 46.19 & 39.33 & 53.17 \\
\hline & & Profesional & & & \\
\hline & & Universitario & 5.08 & 2.64 & 8.82 \\
\hline
\end{tabular}




\begin{tabular}{|c|c|c|c|c|c|}
\hline \multirow{7}{*}{ Perfil ocupacional } & & $\begin{array}{l}\text { Operadores de } \\
\text { instalaciones } \\
\text { y máquinas y } \\
\text { ensambladores }\end{array}$ & 4.57 & 2.28 & 8.17 \\
\hline & $\begin{array}{l}\text { Categórica } \\
\text { Nominal }\end{array}$ & $\begin{array}{l}\text { Personal admi- } \\
\text { nistrativo, servi- } \\
\text { cio y comercio }\end{array}$ & 6.09 & 3.37 & 10.09 \\
\hline & & $\begin{array}{l}\text { Técnicos y Pro- } \\
\text { fesionales de } \\
\text { nivel medio }\end{array}$ & 29.95 & 23.88 & 36.60 \\
\hline & & Otros & 8.63 & 5.31 & 13.16 \\
\hline & & Ninguno & 16.24 & 11.60 & 21.87 \\
\hline & & $\mathrm{Ns} / \mathrm{nr}$ & 19.80 & 14.70 & 25.78 \\
\hline & & 1-Nunca & 14.72 & 10.30 & 20.17 \\
\hline \multirow{4}{*}{ Pasión } & $\begin{array}{l}\text { Categórica } \\
\text { Ordinal }\end{array}$ & 2-Pocas veces & 7.61 & 4.52 & 11.94 \\
\hline & & 3-Regularmente & 14.21 & 9.87 & 19.60 \\
\hline & & 4-Casi Siempre & 34.52 & 28.14 & 41.34 \\
\hline & & 5-Siempre & 42.64 & 35.88 & 49.61 \\
\hline \multirow{5}{*}{ Visión } & & 1-Nunca & 3.05 & 1.28 & 6.17 \\
\hline & $\begin{array}{l}\text { Categórica } \\
\text { Ordinal }\end{array}$ & 2-Pocas veces & 10.15 & 6.52 & 14.95 \\
\hline & & 3-Regularmente & 18.27 & 13.36 & 24.11 \\
\hline & & 4-Casi Siempre & 40.61 & 33.93 & 47.56 \\
\hline & & 5-Siempre & 27.92 & 22.01 & 34.47 \\
\hline \multirow{5}{*}{$\begin{array}{l}\text { Capacidad de } \\
\text { Aprendizaje }\end{array}$} & & 1-Nunca & 1.52 & 0.43 & 4.01 \\
\hline & $\begin{array}{l}\text { Categórica } \\
\text { Ordinal }\end{array}$ & 2-Pocas veces & 5.08 & 2.64 & 8.82 \\
\hline & & 3-Regularmente & 14.21 & 9.87 & 19.60 \\
\hline & & 4-Casi Siempre & 40.61 & 33.93 & 47.56 \\
\hline & & 5-Siempre & 38.58 & 31.99 & 45.50 \\
\hline
\end{tabular}




\begin{tabular}{|c|c|c|c|c|c|}
\hline & & 1-Nunca & 1.02 & 0.21 & 3.22 \\
\hline \multirow{4}{*}{$\begin{array}{l}\text { Logro de resulta- } \\
\text { dos }\end{array}$} & Categórica & 2-Pocas veces & 4.57 & 2.28 & 8.17 \\
\hline & Ordinal & & & & \\
\hline & & 3-Regularmente & 15.74 & 11.17 & 21.30 \\
\hline & & 4-Casi Siempre & 43.15 & 36.37 & 50.12 \\
\hline \multirow{8}{*}{ Determinación } & & 5-Siempre & 35.53 & 29.10 & 42.39 \\
\hline & & 1-Nunca & 1.52 & 0.43 & 4.01 \\
\hline & Categórica & 2-Pocas veces & 10.15 & 6.52 & 14.95 \\
\hline & Ordinal & & & & \\
\hline & & 3-Regularmente & 22.34 & 16.95 & 28.53 \\
\hline & & 4-Casi Siempre & 43.65 & 36.86 & 50.63 \\
\hline & & 5-Siempre & 22.34 & 16.95 & 28.53 \\
\hline & & 1-Nunca & 7.11 & 4.13 & 11.33 \\
\hline \multirow{4}{*}{$\begin{array}{l}\text { Creatividad e In- } \\
\text { novación }\end{array}$} & Categórica & 2-Pocas veces & 11.17 & 7.34 & 16.13 \\
\hline & Ordinal & 3-Regularmente & 19.80 & 14.70 & 25.78 \\
\hline & & 4-Casi Siempre & 45.18 & 38.34 & 52.16 \\
\hline & & 5-Siempre & 16.75 & 12.04 & 22.43 \\
\hline \multirow{7}{*}{ Persistencia } & & 1-Nunca & & & \\
\hline & Categórica & 2-Pocas veces & 3.55 & 1.60 & 6.85 \\
\hline & Ordinal & & & & \\
\hline & & 3-Regularmente & 15.23 & 10.73 & 20.74 \\
\hline & & 4-Casi Siempre & 36.55 & 30.06 & 43.43 \\
\hline & & 5-Siempre & 44.67 & 37.85 & 51.65 \\
\hline & & 1-Nunca & 6.09 & 3.37 & 10.09 \\
\hline \multirow{5}{*}{ Trabajo en equipo } & Categórica & 2-Pocas veces & 12.18 & 8.17 & 17.29 \\
\hline & Ordinal & & & & \\
\hline & & 3-Regularmente & 19.29 & 14.25 & 25.22 \\
\hline & & 4-Casi Siempre & 43.15 & 36.37 & 50.12 \\
\hline & & 5-Siempre & 19.29 & 14.25 & 25.22 \\
\hline \multirow{6}{*}{ Liderazgo } & & 1-Nunca & 1.02 & 0.21 & 3.22 \\
\hline & Categórica & 2-Pocas veces & 7.61 & 4.52 & 11.94 \\
\hline & Ordinal & & & & \\
\hline & & 3-Regularmente & 16.24 & 11.60 & 21.87 \\
\hline & & 4-Casi Siempre & 46.19 & 39.33 & 53.17 \\
\hline & & 5-Siempre & 28.93 & 22.94 & 35.54 \\
\hline
\end{tabular}




\begin{tabular}{|c|c|c|c|c|c|}
\hline \multirow{6}{*}{ Autoestima } & & 1-Nunca & 2.54 & 0.97 & 5.47 \\
\hline & Categórica & 2-Pocas veces & 5.58 & 3.00 & 9.45 \\
\hline & Ordinal & & & & \\
\hline & & 3-Regularmente & 13.20 & 9.02 & 18.45 \\
\hline & & 4-Casi Siempre & 34.52 & 28.14 & 41.34 \\
\hline & & 5-Siempre & 44.16 & 37.36 & 51.14 \\
\hline \multirow{6}{*}{ Asume Riesgos } & & 1-Nunca & 3.55 & 1.60 & 6.85 \\
\hline & Categórica & 2-Pocas veces & 16.75 & 12.04 & 22.43 \\
\hline & Ordinal & & & & \\
\hline & & 3-Regularmente & 18.27 & 13.36 & 24.11 \\
\hline & & 4-Casi Siempre & 40.10 & 33.44 & 47.05 \\
\hline & & 5-Siempre & 21.32 & 16.04 & 27.43 \\
\hline \multirow{5}{*}{ Organización } & & 1-Nunca & 1.52 & 0.43 & 4.01 \\
\hline & & 2-Pocas veces & 4.57 & 2.28 & 8.17 \\
\hline & & 3-Regularmente & 20.81 & 15.59 & 26.88 \\
\hline & & 4-Casi Siempre & 50.76 & 43.81 & 57.69 \\
\hline & & 5-Siempre & 22.34 & 16.95 & 28.53 \\
\hline
\end{tabular}

Fuente: Elaboración propia

La evaluación de fiabilidad del instrumento de medida se realizó mediante el método basado en el estadístico alfa de Cronbach, presentando un alto nivel de consistencia interna de la escala alfa de Cronbach de .839 en 12 elementos (Tabla 2).

Con la finalidad de describir, cuantificar y determinar las relaciones entre las variables predictores y la variable dependiente y determinar la influencia o importancia de estas relaciones sobre la variable dependiente, se aplicó la técnica estadística de análisis multivariante: regresión categórica con escalamiento óptimo (CatReg), la cual permite transformar las variables categóricas originales mediante la asignación de cuantificaciones numéricas a las categorías, obteniéndose una regresión lineal óptima para las variables transformadas (Van der Kooij y Meulman, 1997; De Leeuw, 2005; Haber, Strickland y Guth, 2001).

Así mismo, la Figura 1 se presenta el gráfico de los residuales que refleja que la mayoría de los valores observados se encuentran cerca de la recta diagonal que representa los valores esperados si la distribución fuese normal. 
Tabla 2

Estadísticas de total de elemento

\begin{tabular}{|c|c|c|c|c|}
\hline & $\begin{array}{l}\text { Media de escala si } \\
\text { el elemento se ha } \\
\text { suprimido }\end{array}$ & $\begin{array}{l}\text { Varianza de } \\
\text { escala si el } \\
\text { elemento se ha } \\
\text { suprimido }\end{array}$ & $\begin{array}{l}\text { Correlación total de } \\
\text { elementos corregida }\end{array}$ & $\begin{array}{c}\text { Alfa de Cronbach si el } \\
\text { elemento se ha supri- } \\
\text { mido }\end{array}$ \\
\hline Pasión & 42.59 & 43.437 & .476 & .829 \\
\hline Visión & 42.89 & 41.936 & .550 & .823 \\
\hline $\begin{array}{l}\text { Capacidad de Apren- } \\
\text { dizaje }\end{array}$ & 42.59 & 43.151 & .535 & .825 \\
\hline Logro de resultados & 42.61 & 42.922 & .589 & .821 \\
\hline Determinación & 42.94 & 42.945 & .526 & .825 \\
\hline $\begin{array}{l}\text { Creatividad e Innova- } \\
\text { ción }\end{array}$ & 43.16 & 44.582 & .317 & .843 \\
\hline Persistencia & 42.47 & 43.934 & .535 & .826 \\
\hline Trabajo en equipo & 43.12 & 42.216 & .488 & .829 \\
\hline Liderazgo & 42.75 & 42.945 & .559 & .823 \\
\hline Autoestima & 42.57 & 42.685 & .519 & .826 \\
\hline Asume Riesgos & 43.10 & 43.112 & .427 & .834 \\
\hline Organización & 42.81 & 43.204 & .583 & .822 \\
\hline
\end{tabular}

Fuente: Elaboración propia

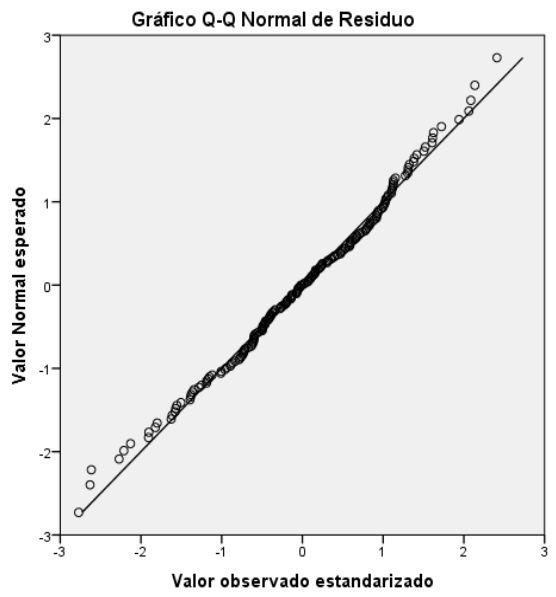

Figura 1. Gráfico Q-Q de las variables transformadas

Fuente: Elaboración propia. 


\section{Resultados y discusión}

Los resultados del modelo CatReg inicial propuesto resulta significativo en el análisis ANOVA $(\mathrm{F}=7.772, p<.05)$ (ver Tabla 3), presentando un coeficiente de determinación ajustado es decir que el $56.80 \%$ de la variabilidad de la variable dependiente "habilidad para detectar y aprovechar oportunidades para la mejora de los resultados" es explicada por todas las variables incluidas en el modelo inicial.

Tabla 3

Resultados modelo inicial

\begin{tabular}{llllll}
\hline & \multicolumn{2}{l}{ Suma de cuadra- } & \multicolumn{2}{l}{ Media } & cua- \\
& dos & gl & drática & F & Sig. \\
\hline Regresión & 128.338 & 38 & 3.377 & 7.772 & 0.000 \\
Residuo & 68.662 & 158 & 0.435 & & \\
Total & 197.000 & 196 & & & \\
\hline
\end{tabular}

Variable dependiente: ¿posee habilidad para detectar y aprovechar oportunidades para la mejora de resultados? Predictores: Grupos de edad, género, estrato socioeconómico, nivel educativo, perfil ocupacional, autoestima, organización, liderazgo, trabajo en equipo, asume riesgos, creatividad e innovación, persistencia, determinación, logro de resultados, capacidad de aprendizaje, pasión, visión.

Fuente: Elaboración propia.

La Tabla 4, presenta las contribuciones parciales a la explicación de la varianza de todos los coeficientes estandarizados de las variables transformadas, indicando que la mayoría de las variables propuestas presentan aportes estadísticamente significativos al modelo $(p<.05)$, con la excepción de las variables 1) edad, 2) género, 3) estrato socioeconómico, 4) nivel educativo, 5) liderazgo, 6) trabajo en equipo, 7) asume riesgos y 8) pasión, $(p>.05)$ en el modelo inicial.

Con el fin de optimizar el modelo, se excluyen secuencialmente las variables que no aportan significancia, proceso que se realiza sistemáticamente bajo el criterio de pasos sucesivos, hasta obtener en conjunto, las variables significativas que explican los resultados de nuevo modelo ajustado, obteniendo un coeficiente de determinación ajustado de (Tabla 5), que indica que aproximadamente el $56.1 \%$ de la varianza de la variable dependiente, "habilidad para detectar y aprovechar oportunidades para la mejora de los resultados", es explicada por la regresión de las variables predictoras significativas $(p<.05)$. 
Tabla 4

Coeficientes de regresión estandarizados para variables transformadas iniciales

\begin{tabular}{|c|c|c|c|c|c|}
\hline \multicolumn{6}{|c|}{ Coeficientes estandarizados } \\
\hline & \multicolumn{3}{|c|}{$\begin{array}{c}\text { Estimación de } \\
\text { bootstrap (1000) de }\end{array}$} & $\mathrm{F}$ & Sig. \\
\hline Edad & -.086 & .057 & 1 & 2.333 & .129 \\
\hline Género & -.010 & .034 & 1 & 0.095 & .759 \\
\hline \multicolumn{6}{|l|}{ Estrato socioeconómico } \\
\hline \multicolumn{6}{|c|}{.040} \\
\hline \multicolumn{6}{|c|}{.041} \\
\hline \multicolumn{6}{|c|}{2} \\
\hline \multicolumn{6}{|c|}{0.957} \\
\hline \multicolumn{6}{|l|}{.386} \\
\hline Nivel educativo & .110 & .088 & 3 & 1.566 & .200 \\
\hline Perfil ocupacional & -.157 & .061 & 5 & 6.610 & .000 \\
\hline Autoestima & -.200 & .067 & 2 & 8.910 & .000 \\
\hline Organización & .323 & .137 & 3 & 5.542 & .001 \\
\hline Liderazgo & .073 & .066 & 3 & 1.206 & .310 \\
\hline Trabajo en equipo & .080 & .042 & 1 & 3.629 & .059 \\
\hline Asume riesgos & -.084 & .064 & 2 & 1.727 & .181 \\
\hline Creatividad e Innovación & .133 & .062 & 2 & 4.567 & .012 \\
\hline Persistencia & .267 & .149 & 2 & 3.200 & .043 \\
\hline Determinación & .170 & .090 & 3 & 3.558 & .016 \\
\hline Logro de resultados & .208 & .138 & 2 & 2.264 & .107 \\
\hline Capacidad de aprendizaje & -.359 & .189 & 2 & 3.627 & .029 \\
\hline Pasión & .113 & .094 & 2 & 1.438 & .240 \\
\hline Visión & .295 & .162 & 2 & 3.317 & .039 \\
\hline \multicolumn{6}{|c|}{ Variable dependiente: Sentido de Oportunidad } \\
\hline Fuente: Elaboración propia & & & & & \\
\hline
\end{tabular}


Tabla 5

Resultados nuevo modelo ajustado

\begin{tabular}{|c|c|c|c|c|c|}
\hline $\mathrm{R}$ múltiple & $R$ cuadrado & $\begin{array}{c}\text { R cuadrado } \\
\text { ajustado }\end{array}$ & $\begin{array}{l}\text { Error de predic- } \\
\text { ción aparente }\end{array}$ & $\begin{array}{l}\text { Error de predic- } \\
\text { ción esperado } \\
\text { Estimación }\end{array}$ & Error estándar \\
\hline .783 & .613 & .561 & .387 & .734 & .187 \\
\hline
\end{tabular}

Fuente: Elaboración propia.

El nuevo resultado del análisis de la varianza presenta la validez del nuevo modelo ajustado. El estimador de la varianza de la regresión (5.250 es mayor que el estimador de la varianza del residuo (.441) y el valor del estadístico de contraste y el nivel crítico asociado ( $\mathrm{F}=11.909$; $p=.000)$, indicando que el ajuste es estadísticamente significativo $(p<.05)$.

La Tabla 6 presenta los coeficientes de regresión parcial estandarizados de las variables transformadas, asociados al modelo ajustado, todos ellos presentan aportes significativos a los valores de la variable modelo $(p<0,05)$, indicando el cambio de las cuantificaciones que cada variable independiente produce en la variable dependiente.

Tabla 6

Coeficientes de regresión parcial estandarizados de las variables transformadas

\begin{tabular}{|c|c|c|c|c|c|}
\hline & Coefici & $\begin{array}{l}\text { estandarizados } \\
\text { Estimación } \\
\text { de bootstrap } \\
\text { (1000) de error } \\
\text { estándar }\end{array}$ & $\mathrm{gl}$ & $\mathrm{F}$ & Sig. \\
\hline Perfil ocupacional & -.187 & .066 & 5 & 8.135 & .000 \\
\hline Autoestima & -.183 & .061 & 2 & 9.060 & .000 \\
\hline Organización & .313 & .136 & 3 & 5.322 & .002 \\
\hline Creatividad e innovación & .128 & .057 & 1 & 4.923 & .028 \\
\hline Persistencia & .276 & .140 & 2 & 3.890 & .022 \\
\hline Determinación & .190 & .088 & 3 & 4.634 & .004 \\
\hline Logro de resultados & .234 & .127 & 3 & 3.363 & .020 \\
\hline Capacidad de aprendizaje & -.344 & .192 & 2 & 3.202 & .043 \\
\hline Visión & .293 & .166 & 2 & 3.119 & .047 \\
\hline
\end{tabular}

Fuente: Elaboración propia. 
En los resultados de la Tabla 6, se observó el cambio de magnitud negativa de las cuantificaciones sobre la variable dependiente de algunas variables independientes como: 1) autoestima,2) perfil ocupacional (es nominal) y 2) capacidad de aprendizaje. Esto podría indicar una percepción de algunas personas consultadas que indistintamente de su perfil ocupacional, de tener o no autoestima, o tener o no capacidad de aprendizaje, consideran que ellos tienen habilidades para detectar y aprovechar oportunidades para la mejora de los resultados, es decir, tienen la creencia de poseer actitud emprendedora por oportunidad, es decir tienen los tres componentes de la actitud: cognitivo (creencia), afectivo (emociones y sentimientos) y comportamental (intención que direcciona la conducta hacia algo) (Robbins y Judge, 2011); actitud enfocada en la transformación las oportunidades de beneficio no aprovechadas en servicios o productos comercializables (Minniti, 2012; Liñan, Fernández-Serrano y Romero, 2013).

Seguidamente, la Tabla 7 muestra las correlaciones de orden cero, donde la mayor correlación entre el predictor transformado (variable dependiente) y la respuesta transformada, se produce para las variables independientes: 1) persistencia; 2) logros de resultados; y 3) determinación. Las medidas de importancia relativa de Pratt, define la importancia de los predictores de forma aditiva, el conjunto de predictores: 1) persistencia; 2) visión; 3) organización y 4) logro de resultados, aportan el 86.4 \% del modelo para explicar la "habilidad para detectar y aprovechar oportunidades para la mejora de los resultados" (variable dependiente).

Tabla 7

Correlaciones de orden cero, parcial e Importancia (variables transformadas)

\begin{tabular}{lcccccc}
\hline & \multicolumn{3}{c}{ Correlaciones } & & & \multicolumn{2}{c}{ Tolerancia } \\
& $\begin{array}{c}\text { Orden } \\
\text { cero }\end{array}$ & Parcial & Part & Importancia & Después de la & Antes de la \\
& & & & transformación & transformación \\
\hline Persistencia & .549 & .317 & .208 & .247 & .565 & .631 \\
Visión & .477 & .351 & .234 & .228 & .636 & .713 \\
Organización & .385 & .361 & .241 & .197 & .592 & .665 \\
Logro de resultados & .503 & .250 & .161 & .192 & .473 & .624 \\
Determinación & .445 & .259 & .167 & .138 & .771 & .676 \\
Perfil ocupacional & -.203 & -.287 & -.186 & .062 & .995 & .971 \\
Creatividad e innovación & .223 & .196 & .124 & .046 & .948 & .895 \\
Autoestima & .083 & -.262 & -.169 & -.025 & .849 & .708 \\
Capacidad de Aprendizaje & .153 & -.365 & -.244 & -.086 & .503 & .724
\end{tabular}

Fuente: Elaboración propia. 
En los hallazgos de la Tabla 7 puede observarse que, de las capacidades exploradas en el estudio, predominan únicamente cinco que explican la variable dependiente. Es decir, las personas consultadas consideran que la persistencia, visión, organización, logro de resultados y determinación son elementos necesarios para el descubrimiento de oportunidades y mejora de resultados. Considerando que los encuestados son personas de los estratos socioeconómicos 1 y 2; $65.48 \%$ jóvenes con edades entre 18 y 25 años; $45.69 \%$ bachilleres y $46.19 \%$ técnicos laborales; $77.6 \%$ son mujeres, se trata de personas que se identifican con el emprendimiento por necesidad. Un hallazgo relevante es la presencia de la cualidad visión de sí mismo como emprendedor, poderoso factor inherente a la persona que la impulsa a seguir participando en la actividad empresarial y que además está relacionado con la autoestima (Farmer, Yao y Kung-Mcintyre, 2011).

Se observan capacidades que los impulsan, pero no necesariamente a tener un negocio propio e independiente, sino como un medio de subsistencia. En estos casos, generalmente está ausente la innovación, entre otros factores, que impulsan el emprendimiento por oportunidad, es decir solo se trata de negocios que tienen un ciclo de vida corto y su impacto en la economía del país es irrelevante (Minniti, Bygrave y Autio, 2006). De igual manera, los resultados revelan que el riesgo no es un factor importante para emprender y administrar un negocio; sin embargo, desde la perspectiva de los inversionistas y cuando se trata de emprendimientos por oportunidad este factor es importante para la toma de decisiones, entrar en nuevos negocios o mercados, así como para el desarrollo de nuevos productos, tal como lo señalan Santillán, Gaona y Hernández (2015).

Es notable destacar la ausencia de la variable creatividad e innovación, cualidad indispensable para un emprendimiento por oportunidad sostenible en el tiempo; así como otras variables como el liderazgo, pasión, capacidad de gestión, aversión al riesgo entre otros que han sido identificadas en el estudio realizado por Quevedo, Izar y Romo (2010).

Según los hallazgos, es importante dotar a las mujeres de conocimiento y herramientas para el fomento del emprendimiento, pues $77.6 \%$ de los consultados son mujeres. Los resultados son contrarios a los hallazgos del estudio de Mitchelmore y Rowley (2013) que encontraron que las competencias empresariales de las mujeres, cuando se trata de emprendimientos por oportunidad son cuatro: 1) competencias personales y de relaciones; 2) habilidad para hacer negocios y competencias gerenciales; 3) competencias empresariales y 4) de relaciones humanas. En términos generales, la formación para el emprendimiento comprende temas como capacitación financiera y contable, así como en las áreas legales y del derecho laboral, es decir, un fomento de competencias financieras (finanzas personales, administración eficiente de recursos) y empresariales (conocimiento del sector donde se desenvuelve la actividad empresarial) (Albornoz et al, 2018). 
El proceso de emprendimiento también demanda incentivos y apoyos que motiven ese espíritu empresarial. Por esa razón, se destacan los incentivos para las nuevas empresas contenidos en la Ley 1429 de Colombia. Así mismo, deben motivarse programas de fomento a la cultura emprendedora desde las políticas locales, regionales y nacionales que permitan fomentar el ecosistema de emprendimiento que abarca desde la formación y capacitación, convocatorias que apoyen a nuevos planes de negocios, programas de fortalecimiento y la creación de unidades de emprendimiento que acompañan el desarrollo de los proyectos de los emprendedores, tal como lo señalan Arias y Giraldo (2011).

\section{Conclusiones}

Los hallazgos encontrados en el estudio revelan que las personas encuestadas perciben tener "habilidad para detectar y aprovechar oportunidades para la mejora de los resultados" a partir de rasgos personales como: persistencia, visión, organización, logro de resultados y determinación, no siendo capacidades suficientes para los negocios que verdaderamente aprovechan oportunidades y son sostenibles en el corto, mediano y largo plazo.

En el análisis se alcanzan a vislumbrar aquellas capacidades inherentes al perfil del emprendedor con las cuales cuenta una muestra de la población de Cúcuta, lo cual constituye una fuente de información o insumo, para delinear estrategias de capacitación para la creación de negocios en las capacidades ausentes como la creatividad e innovación, asumir riesgos, liderazgo y capacidad de gestión empresarial. Según los hallazgos, es indispensable dotar a las mujeres de conocimiento y herramientas para el fomento del emprendimiento, pues $77.6 \%$ de los consultados son mujeres.

Ciertamente, el proceso de emprendimiento depende de la percepción de las personas acerca de sus capacidades y su proyecto de vida. Es por ello, que la capacitación y conocimiento del emprendedor, es un factor importante para que las empresas que ellos generen se alineen a emprendimientos por oportunidad que contribuyan positivamente con la economía del país. Ese conocimiento del emprendedor unido con capital, tecnología y recursos humanos permite el desarrollo de una economía emprendedora y generadora de empleo formal.

Los hallazgos revelan que las capacidades de los emprendedores que se destacan son propias de emprendimientos por necesidad, dado que las capacidades como creatividad e innovación, liderazgo, capacidad para arriesgarse y trabajo en equipo; indispensables en el perfil de emprendedores por oportunidad no explican la variable dependiente: "la percepción de la habilidad para detectar y aprovechar oportunidades para la mejora de los resultados". Estos resultados no distan de la realidad del entorno de una ciudad de Cúcuta donde fue aplicada la encuesta a 197 personas pertenecientes a la BoP. La ciudad de Cúcuta es la capital 
del Departamento Norte de Santander, ubicado en la frontera colombo-venezolana, siendo una región donde existen dinámicas económicas, sociales y culturales caracterizadas por la existencia de una economía formal y legal versus una economía informal, ilegal, más lucrativa y atractiva, basada en el comercio informal, contrabando de gasolina y mercancías, así como el negocio del narcotráfico (Albornoz et al, 2019).

Tampoco pueden desconocerse las actuales cifras de desempleo $(15,5 \%) 5$ puntos porcentuales por encima de la tasa de desempleo nacional; e informalidad (70,3\%); 23,4 puntos porcentuales por encima de la tasa de informalidad de las 13 ciudades y áreas metropolitana de Colombia (junio de 2019) (DANE, 2019). También debe considerarse que en este contexto de frontera, existen municipios donde el conflicto armado colombiano aún permanece, existen grupos armados al margen de la ley que operan y controlan el territorio, y además están vinculados a las dinámicas económicas ilegales. Por ello continúa el desplazamiento interno de colombianos, que se mueven desde municipios en conflicto hacia ciudades como Cúcuta (Morffe, Albornoz-Arias y Mazuera-Arias, 2019). Por lo tanto, en este contexto confluyen la población desplazada por el conflicto interno colombiano; migrantes venezolanos en tránsito, población de frontera que realiza movilidad pendular, migrantes regulares e irregulares; colombianos retornados desde Venezuela y la población local.

Frente a los indicadores de desempleo e informalidad antes referidos, se hace necesario que el gobierno local y regional, consideren en sus planes de gobierno, la superación del desempleo e informalidad a través de políticas o iniciativas de fomento al emprendimiento en la ciudad de Cúcuta y su área metropolitana. Para ello, es fundamental que se realice un diagnóstico para sincerar el volumen de trabajadores dispuestos a trabajar, tomando en cuenta tanto a la población local, como colombianos retornados de Venezuela, desplazados y migrantes (oferta laboral) con relación a la cantidad de empresas o empleadores dispuestos a contratarlos (demanda laboral). Seguidamente, deben generarse las condiciones y mecanismos para la inclusión laboral formal y propiciarse las iniciativas de generación de empleo y autoempleo formal a través del fomento del emprendimiento, que en este contexto de frontera implica: 1) campaña de sensibilización donde el Estado, universidad y medios de comunicación sensibilicen a la población sobre la economía formal, la cultura tributaria; así como también que el hecho de emprender forme parte de un proyecto de vida laboral (Kirby, 2004) y 2) formación para el emprendimiento donde se tomen en cuenta la percepción del potencial emprendedor de sentir la capacidad frente a retos (autoeficacia), perspectivas que se tengan sobre lo que se va a emprender (expectativas) y metas planteadas (Bandura, 1991, 1989, 1986); pudiendo ser el formato de este tipo de educación de tres formas: 1) formal e institucionalizada y planificada por instituciones públicas o privadas; 2) no formal realizada fuera del sistema formal, abarcando aprendizajes dirigidos a adultos a través de cursos, talleres, congresos, conferencias, formación para el trabajo y/o educación a distancia; 3) informal, 
no institucionalizada a través de servicios educativos no académicos y la educación familiar (Formichella, 2008).

Finalmente, queda planteada una propuesta de investigación que permite profundizar en el estudio y análisis de la conducta y las actitudes con relación a la actividad empresarial en la población de la BoP, es decir, abordar el estudio desde la Teoría del Comportamiento Planificado que relaciona la fuerza de la intención como un antecedente inmediato del comportamiento; pues es un mecanismo para descubrir los factores motivacionales que inciden en el comportamiento y en conocer la cantidad de esfuerzo está dispuesto a ejercer dentro de la conducta emprendedora. De esta manera, es posible obtener resultados que permitan a entidades gubernamentales y no gubernamentales, tomar decisiones en materia de intervención, para fortalecer emprendimientos planificados y sostenibles, que generen empleo formal.

\section{Referencias}

Achig, M. F. H., Achig, M. D. R. H., Molestina, M. E. C., Ortega, J. L. C., Vera, F. E. G., y Ortiz, F. X. M. (2018). Competencias emprendedoras en las universidades. Revista Órbita Pedagógica, 5(1), 1-8.

Alaminos, A., y Castejón, J. L. (2006). Elaboración, análisis e interpretación de encuestas, cuestionarios y escalas de opinión. Universidad de Alicante, Alicante: Editorial Marfil S.A.

Albornoz-Arias, N., Acevedo-Gelves, L., Nava Sarmiento, E., Jaimes Tolosa, L., y Díaz Riatiga, J. (2018). Perfil empresarial de estudiantes de Administración de Empresas de la Universidad Simón Bolívar, Cúcuta, Colombia. En N. Albornoz-Arias., L.K. Acevedo-Gelves. y J.F. Espinosa-Castro. (Ed.), La internacionalización de las empresas versus el emprendimiento y la innovación. (pp.153-179). Cúcuta, Colombia: Ediciones Universidad Simón Bolívar.

Albornoz-Arias, N., Mazuera-Arias, R., Millán-Vázquez de la Torre, M. G., y Briceño-León, R. (2019). Los pactos sociales y el contrabando en la frontera colombo-venezolana. Convergencia, 26(81).

Arias, A.C.M., y Giraldo, B.D. P. (2011). Condiciones para la generación de emprendimientos en Medellín y su Área Metropolitana. Revista Virtual Universidad Católica del Norte, 1(34), 138-161.

Azevedo, V., Baigun, A., Bouillon, C. P., Duke, D., y Gallardo Montoya, M. L. (2015). Un mercado creciente de US\$750 mil millones: Descubriendo oportunidades en la base de la pirámide en América Latina y el Caribe. Banco Interamericano de Desarrollo. Disponible en: https://publications.iadb.org/bitstream/handle/11319/6992/Un_Mercado_Creciente_de_US\%24750_mil_millones.pdf?sequence=7yisAllowed=y (Consultado: 21/10/2017).

Bandura, A. (1986). Social foundations of thought and action: Asocial cognitive theory. Englewood Cliffs, NJ: Prentice-Hall.

Bandura, A. (1989). Human agency in social cognitive theory. American Psychologist, 44(9), 1175- 1184.

Bandura, A. (1991). Human agency: The rhetoric and the reality. American Psychologist, 46, 157-162.

Barrera M., S. P. (2012). Análisis del campo del emprendimiento desde un estudio de casos. Revista Ciencias Estratégicas, 20(27), 133-145.

Carreño, M. T., Albornoz, N. C., Mazuera, R., Cuberos, M. A., y Vivas, M. (2018). Formación para el emprendimiento en el gobierno electrónico en Países de la Alianza del Pacífico. Revista ESPACIOS, 39(16), 32-50.

Castellanos, D.O. F., Chávez, P.R.D., y Jiménez, H.C. N. (2003). Propuesta de formación en liderazgo y emprendimiento. Innovar, 13(22), 145-156. 
Contreras V.J. C., Wilches D.S.Y., Delgado R.M., y Cerda C.M. (2016). Mercado base de pirámide urbano y rural en Norte de Santander, Colombia. Revista Venezolana de Gerencia, 21(76), 709-730.

DANE. (2018). Departamento Administrativo Nacional de Estadísticas. Demografía y Población. Disponible en: https://www.dane.gov.co/index.php/estadisticas-por-tema/demografia-y-poblacion (Consultado: 13/05/2018)

DANE (2019). Gran encuesta integrada de hogares (GEIH) Mercado laboral. Disponible en: https://www.dane. gov.co/index.php/estadisticas-por-tema/mercado-laboral/empleo-y-desempleo\#2014 (Consultado: 12/09/2019)

De Leeuw, J. (2005). Multivariate analysis with optimal scaling. Department of Statistics, UCLA. Available from: http://repositories.cdlib.org/uclastat/papers/2005103002/ (Consultado: 12/03/2017).

Duarte, T., y Tibana, M. R. (2009). Emprendimiento, una opción para el desarrollo. Scientia et technica, 3(43), 326-331.

Eijdenberg, E. L., and Masurel, E. (2013). Entrepreneurial motivation in a least developed country: push factors and pull factors among mses in UGANDA, Journal of Enterprising Culture, 21(1), 19-43. https://doi.org/10.1142/ S0218495813500027

Farmer, S. M., Yao, X., and Kung-Mcintyre, K. (2011). The Behavioral Impact of Entrepreneur Identity Aspiration and Prior Entrepreneurial Experience. Entrepreneurship Theory And Practice, 35(2), 245-273. https://doi. org/10.1111/j.1540-6520.2009.00358.x

Ferkany, M. (2008). The educational importance of self-esteem. Journal of Philosophy of Education, 42(1), 119132.

Formichella, M. (2008) Educación y trabajo independiente: Un aporte a la evidencia empírica. Estudios de Economía Aplicada, 26(3), 115-139.

Freire, A. (2005). Pasión por emprender: de la idea a la cruda realidad. Editorial Norma.

Gradl, C., and Knobloch, C. (2010). Inclusive business guide: how to develop business and fight poverty. Berlin: Endeva. Disponible en: http://www.endeva.org/wp-content/uploads/2014/11/IBG_final.pdf (Consultado: 21/10/2017).

Grant-Halvorson, H. (2012). Nine things successful people do differently. Gildan Media Corporation.

Haber, L., Strickland, J. A., and Guth, D. J. (2000). Categorical regression analysis of toxicity data. Comments on toxicology, 7(5-6), 437-452.

Hammond, A., Kramer, W. J., Katz, R; Tran, J., and Walker, C. (2007). The Next 4 Billion: Marketl Size and Business Strategy at the Base of the Pyramid. Pennsylvania: World Resources Institute, Disponible en: http://www. wri.org/publication/the-next-4-billion. (Consultado: 21/10/2017).

Hughes, M., and Morgan, R. E. (2007). Deconstructing the relationship between entre-preneurial orientation and business performance at the embryonic stage of firmgrowth. Industrial Marketing Management, 36, 651-661. https://doi.org/10.1016/j.indmarman.2006.04.003

Kirby, D. A. (2004). Entrepreneurship education: can business school meet the challenge? Education + Training, 46(8/9), 510-519.

Liñán, F., Fernández-Serrano, J., and Romero I. (2013). Necessity and opportunity entrepreneurship: The mediating effect of culture. Revista de Economía Mundial, 33, 21-47.

López, W., Montilla, M., y Briceño, M. (2007). Rasgos determinantes de las aptitudes emprendedoras que forman el perfil de los estudiantes de contaduría pública. Actualidad contable FACES, 10(14), 80-94

Lumpkin, G. T., and Dess, G. G. (1996). Clarifying the entrepreneurial orientationconstruct and linking it to performance. Academy of Management Review, 21(1),135-172.

Martínez-Caro, E., y Cegarra-Navarro, J. G. (2012). El desarrollo de competencias transversales mediante proyectos de emprendimiento en el marco de una asignatura de dirección de operaciones (generic competencies development through entrepreneurship projects in the context of an operations management course). Working Papers on Operations Management, 3(2), 9-13. 
Marulanda V. F. Á., Montoya R. I. A., y Vélez R. J. M. (2014). Teorías motivacionales en el estudio del emprendimiento, Pensamiento y gestión, (36), 204-236. http://dx.doi.org/10.14482/pege.36.5571

Mc Clelland, D. (1961). The Achieving Society. New York: A Free Press Paperback.

Minniti, M. (2012). El emprendimiento y el crecimiento económico de las naciones. Economía industrial, 383, 23-30.

Minniti, M., Bygrave, W. D., and Autio, E. (2006). 2005 Global Entrepreneurship Monitor Report. London: Babson Park, MA: London Business School and Babson College.

Mitchelmore, S., and Rowley, J. (2013). Entrepreneurial competencies of women entrepreneurs pursuing business growth. Journal of small business and enterprise development, 20(1), 125-142.

Morffe Peraza, M.A., Albornoz-Arias, N., Mazuera-Arias, R. (2019). El rostro de la violencia: el posconflicto colombiano y su impacto en la frontera colombo venezolana (Apure-Arauca, Táchira - Norte de Santander). San Cristóbal, Venezuela: Observatorio de Investigaciones Sociales en Frontera. Disponible en: https://jesuitas. lat/attachments/article/1438/El\%20Rostro\%20de\%20la\%20Violencia\%20posconflicto\%20colombiano\%20 Mayo\%202019.pdf (Consultado: 15/07/2019).

Mutis, J., y Ricart, J. E. (2008). Innovación en modelos de negocio: La Base de la Pirámide como campo de experimentación. Universia Business Review, (18), 10-27.

Oosterbeek, H., Van Praag, M., and IJsselstein A. (2008). The Impact of Entrepreneurship Education on Entrepreneurship Competencies and Intentions: An Evaluation of the Junior Achievement Student Mini-Company Program. Tinbergen Institute Discussion Paper No. TI 2008-038/3. http://dx.doi.org/10.2139/ssrn.1118251

Osorio T. F. F, Gálvez A. E. J., y Murillo V.G. (2010). La estrategia y el emprendedor: diversas perspectivas para el análisis. Cuadernos de Administración (Universidad del Valle), (43), 65-80.

Pineda-Escobar, M. A. (2015). Negocios y sostenibilidad en la Base de la Pirámide. Punto de Vista, 5(9), 97-116. doi:10.1108/S2051-5030(2013)0000012009

PNUD (2010). Programa de las Naciones Unidas para el Desarrollo. Crecimiento de mercados inclusivos: estrategias empresariales para la superación de la pobreza y la exclusión en Colombia. Bogotá: PNUD. Disponible en: http://afecolombia.org/Portals/0/Publicaciones/13_Desarrollo/libro_final_final_baja_.pdf, (Consultado: 21/10/2017).

Prahalad, C. K. (2005). La oportunidad de negocios en la base de la pirámide. Buenos Aires: Norma.

Quevedo, L. M., Izar, J. M., y Romo, L. (2010). Factores endógenos y exógenos de mujeres y hombres emprendedores de España, Estados Unidos y México. Investigación y Ciencia, 18(46), 57-63.

Recalde L. M., Villota G. L. R., y Flores J. M. P. (2017). Emprendedores como creadores de riqueza y desarrollo regional. Revista Publicando, 3(9), 564-578.

RedE América (2013). II Foro Base Internacional por el desarrollo de la base en América Latina y el Caribe. Disponible en: http://www.redeamerica.org/Transformadores/Experiencias-detalle/ArtMID/2712/ArticleID/373/ II-Foro-Base-Internacional-por-el-desarrollo-de-la-base-en-Am233rica-Latina-y-el-Caribe, （Consultado: 21/10/2017).

Ricoy L., C. (2006). Contribución sobre los paradigmas de investigación. Educação Revista do Centro de Educação, (31)1, 11-22.

Ries, E. (2011). The Lean Startup: How Constant Innovation Creates Radically Successful Business. Lloc de publicació: Londres. Portfolio Penguin.

Robbins, S. (1999). Comportamiento organizacional, teoría y práctica. (3ra Ed.). México: Prentice Hall Hispanoamericana S.A.

Robbins, S. P., y Judge, T. A. (2011). Organizational behavior. New Yersey: Pearson Prentice Hall.

Rodrigo-Alarcón, J., Parra-Requena, G., y García-Villaverde, P. M. (2014). Efectividad de la orientación emprendedora: el papel del capital social y las capacidades. Investigaciones Europeas de Dirección y Economía de la empresa, 20(3), 131-139.

Rodríguez, C., y Caño, A. (2012). Autoestima en la adolescencia: análisis y estrategias de intervención. International Journal of Psychology and Psychological Therapy, 12(3), 389-403. 
Santillán, S. R. J., Gaona, D. E., y Hernández, P. N. A. (2015). El perfil del emprendedor que apoyan los fondos de capital privado/capital emprendedor en México. Contaduría y administración, 60, 149-174.

Sarasvathy, S. (2001). Causation and Effectuation: Toward a Theoretical Shift from Economic Inevitability to Entrepreneurial Contingency. Academy of Management Review, 26(2), 243-263. https://doi.org/10.5465/ amr.2001.4378020

Schumpeter, J. A. (1961). Entrepreneurial profit. In The theory of economic development (pp. 128-156). Cambridge: Harvard University Press.

Schuster, T., and Holtbrügge, D. (2012). Market entry of multinational companies in markets at the bottom of the pyramid: A learning perspective. International Business Review, 21(5), 817-830. https://doi.org/10.1016/j. ibusrev.2011.09.007

Simanis, E., and Hart, S. (2009). Innovation from the inside out. Sustainability y Innovation MIT Sloan Management Review, Summer, 77-86.

Van Der Kooij A.J. (2007). Prediction accuracy and stability of regression with optimal scaling transformations. Child y Family Studies and Data Theory (AGP-D), Department of Education and Child Studies, Faculty of Social and Behavioural Sciences, Leiden University.

Van der Veen, M., and Wakkee, I. (2002). Understanding the entrepreneurial process. ARPENT: Annual Review of Progress in Entrepreneurship, 2, 114-152. 